\title{
Non-uniqueness
}

\section{in a free boundary problem}

Björn Bennewitz

\begin{abstract}
We show that a result of Lewis and Vogel on uniqueness in a free boundary problem for the $p$-Laplace operator is sharp in two dimensions.
\end{abstract}

\section{Introduction}

Denote points in Euclidean 2 space $\mathbb{R}^{2}$ by $x=\left(x_{1}, x_{2}\right)$. Let $\langle\cdot, \cdot\rangle$ be the standard inner product on $\mathbb{R}^{2}$ and let $|x|=\langle x, x\rangle^{1 / 2}$ be the Euclidean norm of $x$. Set $B(x, r)=\left\{y \in \mathbb{R}^{2}:|x-y|<r\right\}$ whenever $x \in \mathbb{R}^{2}$ and $r>0$. Let $d x$ denote Lebesgue measure on $\mathbb{R}^{2}$ and define $k$ dimensional Hausdorff measure, in $\mathbb{R}^{2}, 0<k \leq 2$, as follows: For fixed $\delta>0$ and $E \subset \mathbb{R}^{2}$, let $L(\delta)=\left\{B\left(x_{i}, r_{i}\right)\right\}$ be such that $E \subset \cup B\left(x_{i}, r_{i}\right)$ and $0<r_{i}<\delta, i=1,2, \ldots$ Set

$$
\phi_{\delta}^{k}(E)=\inf _{L(\delta)}\left(\sum \alpha(k) r_{i}^{k}\right)
$$

where $\alpha(k)$ denotes the volume of the unit ball in $\mathbb{R}^{k}$. Then

$$
H^{k}(E)=\lim _{\delta \rightarrow 0} \phi_{\delta}^{k}(E), \quad 0<k \leq 2
$$

If $O$ is open and $1 \leq q \leq \infty$, let $W^{1, q}(O)$ be the space of equivalence classes of functions $u$ with distributional gradient $\nabla u=\left(u_{x_{1}}, u_{x_{2}}\right)$, both of which are $q$ th power integrable on $O$. Let

$$
\|u\|_{1, q}=\|u\|_{q}+\|\nabla u\|_{q}
$$

be the norm in $W^{1, q}(O)$ where $\|\cdot\|_{q}$ denotes the usual Lebesgue $q$ norm in $O$. Let $C_{0}^{\infty}(O)$ be the space of infinitely differentiable functions with 
compact support in $O$ and let $W_{0}^{1, q}(O)$ be the closure of $C_{0}^{\infty}(O)$ in the norm of $W^{1, q}(O)$. Let $\Omega$ be a domain (i. e. an open connected set) and suppose that the boundary of $\Omega$ (denoted $\partial \Omega$ ) is bounded and non empty. Let $N$ be a neighborhood of $\partial \Omega, p$ fixed, $1<p<\infty$ and $u$ a positive weak solution to the $p$ Laplace differential equation in $\Omega \cap N$. That is $u \in W^{1, p}(\Omega \cap N)$ and

$$
\int|\nabla u|^{p-2}\langle\nabla u, \nabla \theta\rangle d x=0
$$

whenever $\theta \in W_{0}^{1, p}(\Omega \cap N)$. Observe that if $u$ is smooth and $\nabla u \neq 0$ in $\Omega \cap N$, then $\nabla \cdot\left(|\nabla u|^{p-2} \nabla u\right) \equiv 0$ where $\nabla \cdot$ denotes divergence. We assume that $u$ has zero boundary values on $\partial \Omega$ in the Sobolev sense. More specifically if $\zeta \in C_{0}^{\infty}(N)$, then $u \zeta \in W_{0}^{1, p}(\Omega \cap N)$. Extend $u$ to $N \backslash \Omega$ by putting $u \equiv 0$ on $N \backslash \Omega$. Then $u \in W^{1, p}(N)$ and it follows from (1.1) as in [10] that there exists a positive finite Borel measure $\mu$ on $\mathbb{R}^{2}$ with support contained in $\partial \Omega$ and the property that

$$
\int|\nabla u|^{p-2}\langle\nabla u, \nabla \phi\rangle d x=-\int \phi d \mu
$$

whenever $\phi \in C_{0}^{\infty}(N)$. We give a proof that $\mu$ exists provided $u$ has a continuous extension to $N$. It suffices to show

$$
F(\phi)=-\int_{N}\left\langle|\nabla u|^{p-2} \nabla u, \nabla \phi\right\rangle d x \geq 0 .
$$

for $\phi \geq 0$. Then the existence follows from the Riesz representation theorem and the basic estimates listed in section 2. To see this let $\phi=((\epsilon+\max (u-$ $\left.\epsilon, 0))^{\eta}-\epsilon^{\eta}\right) \psi$ where $\psi \in C_{0}^{\infty}(B(z, r))$ and $\psi=1$ on $B(z, r / 2)$ and supp $\psi \subset$ $B(z, r)$ for some $z \in \partial \Omega$. Then supp $\phi \subset \Omega$ so we get

$$
\begin{aligned}
0= & \int_{N}\left\langle|\nabla u|^{p-2} \nabla u, \nabla \phi\right\rangle d x \\
= & \int_{u \geq \epsilon} \eta(\epsilon+\max (u-\epsilon, 0))^{\eta-1}|\nabla u|^{p} \psi d x \\
& +\int_{N}\left((\epsilon+\max (u-\epsilon, 0))^{\eta}-\epsilon^{\eta}\right)|\nabla u|^{p-2}\langle\nabla \psi, \nabla u\rangle d x
\end{aligned}
$$

Note that

$$
\eta \int_{u \geq \epsilon}(\epsilon+\max (u-\epsilon, 0))^{\eta-1}|\nabla u|^{p} \psi d x \geq 0
$$

so

$$
0 \geq \int_{N}\left((\epsilon+\max (u-\epsilon, 0))^{\eta}-\epsilon^{\eta}\right)|\nabla u|^{p-2}\langle\nabla \psi, \nabla u\rangle d x
$$


Suppose $r$ is so small that $u<1$ in $B(z, r)$. Then

$$
\left.\mid(\epsilon+\max (u-\epsilon, 0))^{\eta}-\epsilon^{\eta}\right)\left.|\nabla u|^{p-2}\langle\nabla \psi, \nabla u\rangle\left|\leq\|\nabla \psi\|_{\infty}\right| \nabla u\right|^{p-1} .
$$

Now $|\nabla u| \in L^{p-1}(\Omega)$ so we can use the dominated convergence theorem to take the limits under the integral sign as $\epsilon$ and $\eta$ go to zero and get $F(\psi) \geq 0$. We can use a partition of unity to reduce the problem to such small $r$ 's. Note that if $\partial \Omega$ is smooth enough then

$$
d \mu=|\nabla u|^{p-1} d H^{n-1}
$$

Let $E$ be a compact set and $G$ an open set containing $E$. For fixed $p$, $1<p<\infty$ set

$$
K_{p}(E, G)=\inf \left\{\int|\nabla \theta|^{p} d x\right\}
$$

where the infimum is taken over all $\theta \in C_{0}^{\infty}(G)$ with $\theta=1$ on $E . K_{p}(E, G)$ is called the $p$-capacity of $E$ relative to $G$.

In [17] Lewis and Vogel consider the following free boundary problem. Given $F \subset \mathbb{R}^{n}$ a compact convex set, $a>0$, and $1<p<\infty$, find a function $u$ defined on a domain $D=D(a, p) \supset F$ with

$$
\begin{gathered}
\nabla \cdot\left(|\nabla u|^{p-2} \nabla u\right)=0 \text { weakly in } D \backslash F, \\
u(x) \rightarrow 1 \text { whenever } x \rightarrow y \in F \\
\text { and } u(x) \rightarrow 0 \text { as } x \rightarrow y \in \partial D, \\
\mu=a^{p-1} H^{n-1} \text { on } \partial D .
\end{gathered}
$$

They prove

Theorem A. Suppose $K_{p}(F, G)>0$ for some open $G \supset F$ and let $D, u, p, a$ be as in (1.5a), (1.5b) and let $\mu$ be the measure corresponding to $u$ as in (1.2). If $\mu$ satisfies (1.5c) and in addition there exists $\beta, 0<\beta<\infty$ and $r_{0}>0$, for which

$$
\mu[B(x, r)] \leq \beta r^{n-1}, \quad 0<r<r_{0}
$$

then $u$ and $D$ are uniquely determined.

Previously Henrot and Shahgholian had considered the classical version of this problem that is the problem obtained by replacing (1.5c) by the condition $|\nabla u(x)| \rightarrow a$ whenever $x \rightarrow y \in \partial D$. In [11] they proved

Theorem B. If $K_{p}(F, G)>0$ for some open $G \supset F$ then there exists a unique $\hat{u}, \hat{D}=\hat{D}(a, p)$ such that (1.5a), (1.5b) are satisfied and $|\nabla u(x)| \rightarrow a$. Moreover $\hat{D}$ is convex with a smooth $\left(C^{\infty}\right)$ boundary. 
In this paper we show that theorem A is sharp in two dimensions, namely

Theorem 1. Suppose $n=2$ and $K_{p}(F, G)>0$ for some open $G \supset F$. If $a>0$ and $1<p<\infty$ there exists a bounded domain $D$ which is not convex, a $p$ harmonic function $u$ and a corresponding measure $\mu$ which satisfy (1.5) but $\mu$ does not satisfy (1.6).

The proof uses the same method as the construction of pseudospheres in [16] to construct a domain which satisfies (1.5) but is not convex and thus is not the same as the domain in [11]. To outline this method let $\Omega$ be a domain and let $u$ be a function which satisfies (1.5a), (1.5b) with $D$ replaced by $\Omega$ and suppose $a=1$. If $p<2$ suppose that $|\nabla u|>1$ on $\partial \Omega$ but if $p>2$ suppose $|\nabla u|<1$ on $\partial \Omega$. For a given small $\epsilon$ we add smooth bumps to $\partial \Omega$ by "pushing out" or "pushing in" along certain surface elements of $\{x \in \partial \Omega:|\nabla u(x)|>1+\epsilon\}$ or $\{x \in \partial \Omega:|\nabla u(x)|<1-\epsilon\}$ depending on whether $p>2$ or $p<2$. In this way we obtain a new domain $\Omega^{\prime} \supset \Omega$ if $p<2$ but $\Omega^{\prime} \subset \Omega$ if $p>2$ and we choose the bumps so that for $\epsilon \leq t \leq 1$

$$
H^{1}\left(\partial \Omega^{\prime}\right) \geq H^{1}(\partial \Omega)+\eta(t) H^{1}\{x:|\nabla u(x)|>1+t\}
$$

if $p<2$ but

$$
H^{1}\left(\partial \Omega^{\prime}\right) \geq H^{1}(\partial \Omega)+\eta(t) H^{1}\{x:|\nabla u(x)|<1-t\}
$$

if $p>2$. Here $\eta$ is a positive function on $] 0, \infty\left[\right.$. Let $u^{\prime}$ be a function in $\Omega^{\prime}$ which satisfies (1.5a), (1.5b) with $D$ replaced by $\Omega^{\prime}$. If $p<2$ then $\Omega \subset \Omega^{\prime}$ and it follows that $u \leq u^{\prime}$ in $\Omega$ and by the maximum principle $\left|\nabla u^{\prime}\right|>1$ on $\partial \Omega \cap \partial \Omega^{\prime}$. In section 3 we prove that $\left|\nabla u^{\prime}\right|>1$ on the bumps. If $p>2$ we get $\left|\nabla u^{\prime}\right|<1$ in the same way. In section 4 we will show that there exists a certain elliptic partial differential equation for which $u^{\prime}$ is a solution and $\log \left|\nabla u^{\prime}\right|$ is a supersolution if $1<p<2$ and a subsolution if $p>2$. Then we use the divergence theorem as in [2] to prove that if $1<p<2$ then

$$
\int_{\partial \Omega^{\prime}}\left|\nabla u^{\prime}\right|^{p-1} \log \left|\nabla u^{\prime}\right| d H^{1} \leq C
$$

and if $p>2$ then

$$
\int_{\partial \Omega^{\prime}}\left|\nabla u^{\prime}\right|^{p-1} \log \left|\nabla u^{\prime}\right| d H^{1} \geq C
$$

where the constant $C$ depends only on $F$. If $1<p<2$ this allows us to control the size of the set where $\left|\nabla u^{\prime}\right|$ is large so that by pushing out and keeping $\left|\nabla u^{\prime}\right|>1$ we in fact keep $\left|\nabla u^{\prime}\right|$ close to 1 for the most part. Likewise if $p>2$ we are able to control the size of the set where $\left|\nabla u^{\prime}\right|$ is close to zero. 
Finally we use (1.7)-(1.10) and induction to construct $D$. We describe the case $p<2$ in detail, the case $p>2$ is similar. Let $D_{0}$ be a domain such that $u_{0}$ satisfies (1.5a) and (1.5b) with $D$ replaced by $D_{0}$ and let $\Omega=D_{0}$. Modify $\Omega$ as above to get $\Omega^{\prime}=D_{1}$ and $u^{\prime}=u_{1}$. If $D_{k}$ has been constructed for $0 \leq k \leq m$ we put $\epsilon_{m}=2^{-m} \epsilon_{0}$ and modify $D_{m}$ to obtain $D_{m+1}$. Set $D=\bigcup_{0}^{\infty} D_{k}$. The construction can be arranged so that $D$ is not convex (see Section 4) which shows that it is not the domain in [17]. To prove (1.5c) we first note

$$
C \geq \int d \mu_{k}=\int_{\partial D_{k}}\left|\nabla u_{k}\right|^{p-1} d H^{1} \geq H^{1}\left(\partial D_{k}\right)
$$

for $k=0,1, \ldots$ because $\mu_{k}\left(\partial D_{k}\right) \leq C$ for some $C$ independent of $k$ (see Section 4). Second, for each $\delta>0$ we have

$$
\lim _{k \rightarrow \infty} H^{1}\left\{x \in \partial D_{k}:\left|\nabla u_{k}(x)\right|>1+\delta\right\}=0
$$

since otherwise (1.7) and iteration would lead to a contradiction to (1.11). Next from (1.9) and the fact that $\left|\nabla u_{k}\right|>1$ on $\partial D_{k}$ we see that for $M>1$ and $k=0,1, \ldots$

$$
\text { (1.13) } \log M \int_{\left\{\left|\nabla u_{k}\right|>M\right\}}\left|\nabla u_{k}\right|^{p-1} d H^{1} \leq \int_{\partial D_{k}}\left|\nabla u_{k}\right|^{p-1} \log \left|\nabla u_{k}\right| d H^{1} \leq C<\infty .
$$

We also show that as $k \rightarrow \infty$

$$
\left.\left.H^{1}\right|_{\partial D_{k}} \rightarrow H^{1}\right|_{\partial D} \text { and } \mu_{k} \rightarrow \mu
$$

weakly as measures on $\mathbb{R}^{2}$ in section 4 . Let $\phi \in C_{0}^{\infty}\left(\mathbb{R}^{2}\right)$ and $\phi \geq 0$. Then we get

$$
\int \phi d \mu_{k}=\int_{\partial D_{k}} \phi\left|\nabla u_{k}\right|^{p-1} d H^{1} \geq \int_{\partial D_{k}} \phi d H^{1} .
$$

To obtain the reverse inequality let $\delta$ be a fixed small number and $M$ be a fixed large number and put

$$
\begin{aligned}
& E_{k}=\left\{x \in \partial D_{k}: 1 \leq\left|\nabla u_{k}(x)\right| \leq 1+\delta\right\} \\
& F_{k}=\left\{x \in \partial D_{k}: 1+\delta<\left|\nabla u_{k}(x)\right| \leq M\right\} \\
& L_{k}=\left\{x \in \partial D_{k}:\left|\nabla u_{k}(x)\right|>M\right\}
\end{aligned}
$$

for $k=0,1, \ldots$. Then

$$
\int \phi d \mu_{k}=\int_{\partial D_{k}} \phi\left|\nabla u_{k}\right|^{p-1} d H^{1}=\int_{E_{k}} \cdots+\int_{F_{k}} \cdots+\int_{L_{k}} \cdots=I_{1}+I_{2}+I_{3} .
$$

It is clear that

$$
\left|I_{1}\right| \leq(1+\delta)^{p-1} \int_{\partial D_{k}} \phi d H^{1}
$$


Also from (1.12) we have

$$
\left|I_{2}\right| \leq M^{p-1}\|\phi\|_{\infty} H^{1}\left\{x \in \partial D_{k}: 1+\delta<\left|\nabla u_{k}\right|\right\} \rightarrow 0
$$

as $k \rightarrow \infty$. Using (1.13) we get

$$
\left|I_{3}\right| \leq\|\phi\|_{\infty} \int_{\left|\nabla u_{k}\right|>M}\left|\nabla u_{k}\right|^{p-1} d H^{1} \leq \frac{C}{\log M}\|\phi\|_{\infty}
$$

Letting $k \rightarrow \infty$ we obtain from the above and (1.14)

$$
\int_{\partial D} \phi d H^{1} \leq \int \phi d \mu \leq(1+\delta)^{p-1} \int_{\partial D} \phi d H^{1}+\frac{C}{\log M}\|\phi\|_{\infty} .
$$

Finally letting $\delta \rightarrow 0$ and $M \rightarrow \infty$ we obtain

$$
\int \phi d \mu=\int_{\partial D} \phi d H^{1}
$$

which is what we wanted to prove. Finally the author would like to thank J. Lewis for pointing out this problem and helpful discussions.

\section{Basic estimates}

A Jordan curve $J$ is said to be a $k$ quasicircle $0<k<1$ if $J=f(\partial B(0,1))$ where $f \in W^{1,2}\left(\mathbb{R}^{2}\right)$ is a homeomorphism of $\mathbb{R}^{2}$ and

$$
\left|f_{\bar{z}}\right| \leq k\left|f_{z}\right|, \quad H^{2} \text { a. e. in } \mathbb{R}^{2} .
$$

Here we use complex notation, $i=\sqrt{-1}, z=x_{1}+i x_{2}, 2 f_{\bar{z}}=f_{x_{1}}+i f_{x_{2}}$, $2 f_{z}=f_{x_{1}}-i f_{x_{2}}$. We call $J$ a quasicircle if $J$ is a $k$ quasicircle for some $0<k<1$. Let $w_{1}, w_{2}$ be distinct points on the Jordan curve $J$ and $J_{1}, J_{2}$ the arcs with endpoints $w_{1}, w_{2}$. Then $J$ is said to satisfy the Ahlfors three point condition if there exists an $1 \leq M<\infty$ such that for all $w_{1}, w_{2} \in J$ we have

$$
\min \left\{\operatorname{diam} J_{1}, \operatorname{diam} J_{2}\right\} \leq M\left|w_{1}-w_{2}\right| .
$$

A Jordan curve $J$ is a quasicircle if and only if it satisfies the Ahlfors three point condition. A domain $\Omega$ is said to be uniform provided there exists $M, 1 \leq M<\infty$ such that if $w_{1}, w_{2} \in \Omega$, then there is a rectifiable curve $\gamma:[0,1] \rightarrow \Omega$ with $\gamma(0)=w_{1}, \gamma(1)=w_{2}$, and

$$
\begin{gathered}
H^{1}(\gamma) \leq M\left|w_{1}-w_{2}\right| \\
\min \left\{H^{1}(\gamma([0, t])), H^{1}(\gamma([t, 1]))\right\} \leq M d(\gamma(t), \partial \Omega)
\end{gathered}
$$

where $d(\underset{\sim}{E}, F)$ denotes the distance between two non-empty sets $E$ and $F$. If $1 \leq \tilde{M}<\infty$ and $\Omega$ is a domain a ball $B(w, r) \subset \Omega$ is said to be $\tilde{M}$ non-tangential if

$$
\tilde{M} r>d(B(w, r), \partial \Omega)>\tilde{M}^{-1} r
$$


If $w_{1}, w_{2} \in \Omega$ a Harnack chain from $w_{1}$ to $w_{2}$ in $\Omega$ is a sequence of $\tilde{M}$ non-tangential balls such that the first ball contains $w_{1}$ the last ball contains $w_{2}$ and consecutive balls intersect. The conditions (2.2) are equivalent to

For any $w \in \partial \Omega, 0<r \leq \operatorname{diam} \Omega$, there exists

$a=a_{r}(w) \in \Omega$ such that $M^{-1} r<|a-r|<r$ and

$d(a, \partial \Omega)>M^{-1} r$

Given $\epsilon>0, w_{1}, w_{2} \in \Omega, d\left(w_{j}, \partial \Omega\right)>\epsilon$ and

$\left|w_{1}-w_{2}\right|<C \epsilon$, there is a Harnack chain from

$w_{1}$ to $w_{2}$ whose length depends on $C$ but not on $\epsilon$.

See [9] for references.

In the sequel $c$ will denote a positive constant $\geq 1$ (not necessarily the same at each occurrence) which may depend only on $p$ unless otherwise stated. In general $c\left(a_{1}, \ldots, a_{n}\right)$ denotes a positive constant $\geq 1$ which may only depend on $p, a_{1}, \ldots, a_{n}$, not necessarily the same at each occurrence. We begin by stating some interior and boundary estimates for $u$ a positive weak solution to the $p$ Laplacian in $B(w, 4 r) \cap \Omega$ with $u=0$ on $\partial \Omega \cap B(w, 4 r)$ when this set is nonempty. In this case we extend $u$ to $B(w, 4 r)$ by putting $u=0$ on $B(w, 4 r) \backslash \Omega$. Let $\max _{B(z, s)} u, \min _{B(z, s)} u$ be the essential supremum and infimum of $u$ on $B(z, s)$ whenever $B(z, s) \subset B(w, 4 r)$.

Lemma 1. Let $u$ be as above. Then

$$
c^{-1} r^{p-2} \int_{B(w, r / 2)}|\nabla u|^{p} d x \leq \max _{B(w, r)} u^{p} \leq c r^{-2} \int_{B(w, 2 r)} u^{p} d x .
$$

If $B(w, 2 r) \subset \Omega$, then

$$
\max _{B(w, r)} u \leq c \min _{B(w, r)} u .
$$

Proof. The first display in Lemma 1 is a standard subsolution estimate while the second display is a standard weak Harnack estimate for positive weak solutions to nonlinear partial differential equations of $p$ Laplacian type (see $[20])$.

Lemma 2. Let $u$ be as in Lemma 1. Then $u$ has a representative in $W^{1, p}(B(w, 4 r) \cap \Omega)$ with Hölder continuous partial derivatives in $B(w, 4 r) \cap$ $\Omega$. That is for some $\sigma=\sigma(p) \in] 0,1[$ we have

$$
\begin{aligned}
c^{-1}\left|\nabla u\left(w_{1}\right)-\nabla u\left(w_{2}\right)\right| & \leq\left(\left|w_{1}-w_{2}\right| / s\right)^{\sigma} \max _{B(z, s)}|\nabla u| \\
& \leq c s^{-1}\left(\left|w_{1}-w_{2}\right| / s\right)^{\sigma} \max _{B(z, 2 s)} u
\end{aligned}
$$

whenever $w_{1}, w_{2} \in B(z, s)$ and $B(z, 4 s) \subset B(w, 4 r) \cap \Omega$. 
Proof. The proof of Lemma 2 can be found in [4], [14] or [21] and in fact is true when $B(w, 4 r) \cap \Omega \subset \mathbb{R}^{n}$. In $\mathbb{R}^{2}$ the best Hölder exponent in Lemma 2 is known when $p>2$ while for $1<p \leq 2$ a solution has continuous second partials (see [12]).

A mapping $h: B(w, 4 r) \cap \Omega \rightarrow \mathbb{R}^{2}$ is said to be quasiregular in $B(w, 4 r) \cap$ $\Omega$ if $h \in W^{1,2}(B(w, 4 r) \cap \Omega)$ and (2.1) holds with $f$ replaced by $h$ in $B(w, 4 r) \cap \Omega$. From a factorization theorem for quasiregular mappings it follows that $h=\tau \circ f$ where $f$ is quasiconformal in $\mathbb{R}^{2}$ and $\tau$ is an analytic function on $f(B(w, 4 r) \cap \Omega)$.

Lemma 3. If $u$ is as in Lemma 1 and $z=x_{1}+i x_{2}$ then $u_{z}$ is quasiregular in $B(w, 4 r) \cap \Omega$ for some $0<k<1$ (depending only on $p$ ) and consequently $\nabla u$ has only isolated zeros in $B(w, 4 r) \cap \Omega$.

Proof. For a proof of quasiregularity see [1], [15]. Since the zeros of an analytic function are isolated it follows from the factorization theorem that the zeros of $\nabla u$ are isolated.

Lemma 4. If $B(w, 4 r) \subset \Omega, \nabla u \neq 0$ in $B(w, 4 r)$ and $\max _{B(w, 2 r)}|\nabla u| \leq$ $\lambda \max _{B(w, r)}|\nabla u|$ then

$$
\max _{B(w, 2 r)}|\nabla u| \leq c(\lambda) \min _{B(w, r)}|\nabla u|
$$

Proof. Note that $v=\log |\nabla u|$ is a weak solution in $B(w, 4 r)$ to the divergence form partial differential equation (see [19])

$$
\sum_{i, j=1}^{2} \frac{\partial}{\partial x_{i}}\left(A_{i j}(x) v_{x_{j}}\right)=0
$$

where the $\left(A_{i j}\right)$ are bounded and uniformly elliptic (with constants depending only on $p$ ). Using Harnacks inequality for positive solutions to partial differential equations of this type (see [20]) applied to $\max _{B(w, 2 r)} v-v$ in $B(w, r)$ we obtain the lemma.

Lemma 5. Let $u$ be as in Lemma 1 and $w \in \partial \Omega$. If $p>2$ there exists $\alpha=\alpha(p) \in] 0,1[$ such that $u$ has a Hölder $\alpha$ continuous representative in $B(w, r)$ (also denoted $u$ ). Moreover if $x, y \in B(w, r)$ then

$$
|u(x)-u(y)| \leq c(|x-y| / r)^{\alpha} \max _{B(w, 2 r)} u .
$$

If $1<p \leq 2$ and $\Omega$ is simply connected, then this inequality is also valid when $1<p \leq 2$ with $\alpha=\alpha(p)$. 
Proof. For $p>2$, Lemma 5 is a consequence of Lemma 1 and Morreys inequality (see [6]). If $1<p \leq 2$ and $\Omega$ is simply connected we deduce from the interior estimates in Lemma 2 that it suffices to consider only the case when $y \in B(w, r) \cap \partial \Omega$. We then show for some $\theta=\theta(p, k), 0<\theta<1$ that

$$
\max _{B(z, \rho / 4)} u \leq \theta \max _{B(z, \rho / 2)} u \quad \text { whenever } 0<\rho<r \text { and } z \in \partial \Omega \cap B(w, r) .
$$

This inequality can then be iterated to get Lemma 5 for $x, y$ as above. To prove (2.4) we use the fact that $B(z, \rho / 4) \cap \partial \Omega$ and $B(z, \rho / 4)$ have comparable $p$ capacities (see [10]) and estimates for subsolutions to elliptic partial differential equations of $p$ Laplacian type (see [8], [15]).

Lemma 6. Let $u, \Omega, w$ be as in Lemma 5. Assume also that $\Omega$ is a uniform domain. Then there exist $c=c(M)$ and $\hat{c}=\hat{c}(M)$ with

$$
\max _{B(w, r / \hat{c})} u \leq c u\left(a_{r / \hat{c}}(w)\right)
$$

where $M$ is as in (2.2) and $a_{r}(w)$ is as in (2.3). Hence

$$
|u(x)-u(y)| \leq c(|x-y| / r)^{\alpha} u\left(a_{r / \hat{c}}(w)\right)
$$

for $x, y \in B(w, r / 2 \hat{c})$.

Proof. The first display in Lemma 6 follows from Harnacks principle in Lemma 1 , Hölder continuity of $u$ in Lemma 5 and the fact that $\Omega$ is a uniform domain and a general argument which can be found in [3]. The second display follows from the first display and Lemma 5

To proceed we consider the following scenario. Let $\Omega$ be a domain such that $\partial \Omega$ is $C^{4}$. Let $w \in \partial \Omega$ and let $u$ be a positive $p$ harmonic function in $\Omega \cap B(w, 2 r)$ and assume that $\Omega \cap B(w, 2 r)$ has only one component. We further assume that $\nabla u \neq 0$ in $\Omega \cap B(w, r)$. We have

Lemma 7. Let $u$ be as above. If $x \in \Omega \cap B(w, r)$ there exists a $c \geq 1$ depending only on $k$ and $p$ such that

$$
c^{-1} d(x, \partial \Omega)^{-1} u(x) \leq|\nabla u(x)| \leq c d(x, \partial \Omega)^{-1} u(x)
$$

where $d(x, \partial \Omega)$ denotes the distance from $x$ to $\partial \Omega$

Proof. Choose $y \in B(x, d(x, \partial \Omega))$ with $u(y)=u(x) / 2$. Apply the mean value theorem of calculus to $u$ restricted to the line segment with endpoints $x, y$. From this and Lemma 6 it follows that there exists a constant $c \geq 4$ 
and $z$ such that $y \in B\left(x,\left(1-c^{-1}\right) d(x, \partial \Omega)\right)$ and $z$ is on the line segment between $x$ and $y$ and

$$
u(x) / 2=|u(x)-u(y)| \leq|\nabla u(z)||x-y| .
$$

Using this inequality and Lemma 2 we see for some positive $\tilde{c}$ that if $t_{1}=$ $\left(1-c^{-1}\right) d(x, \partial \Omega), t_{2}=\left(1-(2 c)^{-1}\right) d(x, \partial \Omega)$ then

$$
\tilde{c}^{-1} u(x) / d(x, \partial \Omega) \leq \max _{B\left(x, t_{1}\right)}|\nabla u| \leq \max _{B\left(x, t_{2}\right)}|\nabla u| \leq \tilde{c} u(x) / d(x, \partial \Omega) .
$$

From (2.5) and Lemma 4 we conclude that Lemma 7 is valid for $u$ at $x$.

Let $\theta$ be a function whose graph is after a rotation and translation $\Omega \cap$ $B(w, r / 2)$ and suppose that the $C^{4}$-norm of $\theta$ is bounded by $c / r$. The condition (3.1) stated in the next section is clearly sufficient. At each point $x \in \partial \Omega \cap B(w, r / 2)$ we can find a tangential ball $B(z, \rho) \subset \Omega \cap B(w, r)$ with $x \in \partial B(z, \rho)$ and radius $\rho>0$ depending only on $\lambda$ and $r$. Let $v$ be the $p$ harmonic function which is zero on $\partial B(z, \rho)$ and $\inf _{\partial B(z, \rho / 2)} u$ on $\partial B(z, \rho / 2)$. Then $v \leq u$ in the annulus $B(z, \rho) \backslash B(z, \rho / 2)$. Therefore

$$
|\nabla u(t)| \geq c^{-1} u(t) d(t, \partial \Omega)^{-1} \geq c^{-1} v(t) d(t, \partial \Omega)^{-1} \geq c^{-1} \inf _{\partial B(z, \rho / 2)} u / \rho
$$

for $t$ in the annulus where we used the fact that $v(x)=A|x-z|^{\frac{p-2}{p-1}}+B$ to compute $\nabla v$. Then by Harnack's inequality we get a lower bound in terms of $\max _{B(w, r)} u$. We can argue in the same way to get an upper bound so that we have

$$
c^{-1} \max _{B(w, r)} u / r \leq|\nabla u|(t) \leq c \max _{B(w, r)} u / r
$$

for $t$ in $B(w, r / 2) \cap \Omega$ and thus $u \in W^{1,2}(B(w, r / 2))$.

Let $\tilde{u}(x)=u(r x+w) / r$. Then $\tilde{u}$ is a solution to the $p$ Laplace equation in $B(0,2) \cap \tilde{\Omega}$ where $\tilde{\Omega}=\left\{x \in \mathbb{R}^{2}: r x+w \in \Omega\right\}$. Let $\Phi$ be a differentiable mapping from $B(0,2)$ to $B(0,2)$ such that 0 is mapped to 0 and ] - 1, 1 [ is mapped to $\partial \tilde{\Omega} \cap B(0,2)$ and $\{(x, y) \in B(0,2): y>0\}$ is mapped to $B(0,2) \cap \tilde{\Omega}$. Define $v=\tilde{u} \circ \Phi$ in $\{(x, y) \in B(0,2): y>0\}$ and let $v(x, y)=0$ in $\{(x, y) \in B(0,2): y<0\}$. Then $v$ satisfies an equation of the form

$$
\nabla \cdot\left(\langle A \nabla v, \nabla v\rangle^{p / 2-1} A \nabla v\right)=0
$$

in $B(0,2)^{+}=B(0,2) \cap\{(x, y) \in B(0,2): y>0\}$ where $A=\left[A_{i j}\right]$ is a symmetric matrix whose coefficients are in $C^{1}$. From our work above it follows that

$$
c^{-1} \max _{B(0,2)} v \leq|\nabla v|(x) \leq c \max _{B(0,2)} v
$$


for $x \in B(0,1)^{+}$where the constant may depend on $\Phi$. If we let $A(x, \xi)=$ $\langle A \xi, \xi\rangle^{p / 2-1} A \xi$ then we have

$$
(|\xi|+|\eta|)^{p-2}|\xi-\eta|^{2} \leq c\langle A(x, \xi)-A(x, \eta), \xi-\eta\rangle
$$

and

$$
\left|\nabla_{x} A(x, \eta)\right| \leq c|\eta|^{p-1}
$$

where $\nabla_{x}$ denotes the gradient with respect to the $x$ variable.

Lemma 8. Let $v$ be as above. Then $v$ has weak derivatives of second order and $v_{x_{1}} \in W^{1,2}(B(0,1 / 2))$ and we have

$$
\int_{B(z, \rho / 2)} \sum_{i=1}^{2}\left|v_{x_{j} x_{i}}\right|^{2} d x \leq \frac{c}{\rho^{2}} \int_{B(z, \rho)}\left|v_{x_{j}}-a\right|^{2} d x+c \rho^{2}\left(\max _{B(0,1)} v\right)^{2}
$$

if $B(z, \rho) \subset B(0,1 / 2)^{+}=\left\{\left(x_{1}, x_{2}\right) \in B(0,1 / 2): x_{2}>0\right\}$ and $a \in \mathbb{R}$. In addition we have

$$
\int_{B(z, \rho / 2)} \sum_{i=1}^{2}\left|v_{x_{1} x_{i}}\right|^{2} d x \leq \frac{c}{\rho^{2}} \int_{B(z, \rho)}\left|v_{x_{1}}\right|^{2} d x+c \rho^{2}\left(\max _{B(0,1)} v\right)^{2}
$$

for any $z \in B(0,1 / 2)$ and $\rho \leq 1 / 4$

Proof. Let

$$
D_{k}^{h} v(x)=\frac{v\left(x+h e_{k}\right)-v(x)}{h}
$$

where $e_{k}$ denotes the $k$-th unit vector. Let $\zeta$ be a smooth function such that $\zeta=1$ on $B(z, \rho / 2), \operatorname{supp} \zeta \subset B(z, \rho)$ and $|\nabla \zeta| \leq c / \rho$ for some constant $c$. Since $v \in W^{1,2}\left(B(0,1)^{+}\right)$and $v=0$ on $\left\{\left(x_{1}, x_{2}\right): x_{2}=0\right\}$ the function $\phi=D_{k}^{-h}\left(\zeta^{2}\left(D_{k}^{h} v-a\right)\right)$ belongs to $W_{0}^{1,2}\left(B(0,1)^{+}\right)$if $B(z, \rho) \subset B(0,1 / 2)^{+}$ and if $a=0$ and $k=1$ we have $\phi \in W_{0}^{1,2}\left(B(0,1)^{+}\right)$for any $z \in B(0,1 / 2)$ and $\rho \leq 1 / 4$. This function is therefore an admissible test function. We obtain

$$
\begin{aligned}
0= & \int\left\langle A(x, \nabla v), \nabla\left(D_{k}^{-h}\left(\zeta^{2}\left(D_{k}^{h} v-a\right)\right)\right)\right\rangle d x \\
= & \int\left\langle D_{k}^{h} A(x, \nabla v), \nabla\left(\zeta^{2}\left(D_{k}^{h} v-a\right)\right)\right\rangle d x \\
= & \int\left\langle\frac{A\left(x+h e_{k}, \nabla v\left(x+h e_{k}\right)\right)-A\left(x+h e_{k}, \nabla v(x)\right)}{h}, \nabla\left(\zeta^{2}\left(D_{k}^{h} v-a\right)\right)\right\rangle d x \\
& +\int\left\langle\frac{A\left(x+h e_{k}, \nabla v(x)\right)-A(x, \nabla v(x))}{h}, \nabla\left(\zeta^{2}\left(D_{k}^{h} v-a\right)\right)\right\rangle d x=\mathrm{I}+\mathrm{II}
\end{aligned}
$$


The first of these integrals is

$$
\begin{aligned}
& \int\left\langle\frac{A\left(x+h e_{k}, \nabla v\left(x+h e_{k}\right)\right)-A\left(x+h e_{k}, \nabla v(x)\right)}{h}, \zeta^{2} D_{k}^{h} \nabla v\right\rangle d x \\
& +\int\left\langle\frac{A\left(x+h e_{k}, \nabla v\left(x+h e_{k}\right)\right)-A\left(x+h e_{k}, \nabla v(x)\right)}{h}, 2 \zeta \nabla \zeta\left(D_{k}^{h} v-a\right)\right\rangle d x
\end{aligned}
$$

The first term in this expression can be bounded below by

$$
c^{-1} \int\left(\left|\nabla v\left(x+h e_{k}\right)\right|+|\nabla v(x)|\right)^{p-2} \zeta^{2}\left|D_{k}^{h} \nabla v\right|^{2} d x
$$

and the second term can be bounded above by

$$
\begin{aligned}
\int\left(\left|\nabla v\left(x+h e_{k}\right)\right|\right. & +|\nabla v(x)|)^{p-2}\left|D_{k}^{h} \nabla v\right||\nabla \zeta|\left|2 \zeta\left(D_{k}^{h} v-a\right)\right| d x \\
\leq & \epsilon \int\left(\left|\nabla v\left(x+h e_{k}\right)\right|+|\nabla v(x)|\right)^{p-2}\left|D_{k}^{h} \nabla v\right|^{2} \zeta^{2} d x \\
& +\frac{c}{\epsilon} \int\left(\left|\nabla v\left(x+h e_{k}\right)\right|+|\nabla v(x)|\right)^{p-2}|\nabla \zeta|^{2}\left|D_{k}^{h} v-a\right|^{2} d x
\end{aligned}
$$

by Youngs inequality. As for II we get

$$
\begin{aligned}
\mathrm{II} & \leq c \int|\nabla v|^{p-1} \zeta^{2}\left|D_{k}^{h} \nabla v\right| d x+c \int|\nabla v|^{p-1}|\zeta||\nabla \zeta|\left|D_{k}^{h} v-a\right| d x \\
& \leq \epsilon \int|\nabla v|^{p-2} \zeta^{2}\left|D_{k}^{h} \nabla v\right|^{2} d x+\frac{c}{\epsilon} \int|\nabla v|^{p} \zeta^{2} d x+\frac{c}{\rho^{2}} \int|\nabla v|^{p-2}\left|D_{k}^{h} v-a\right|^{2} d x .
\end{aligned}
$$

Choosing $\epsilon$ small enough and using (2.8) to estimate $|\nabla v|^{p-2}$ we get

$$
\int_{B(z, \rho / 2)}\left|D_{k}^{h} \nabla v\right|^{2} d x \leq \frac{c}{\rho^{2}} \int_{B(z, \rho)}\left|v_{x_{k}}-a\right|^{2} d x+\rho^{2}\left(\max _{B(0,1)} v\right)^{2} .
$$

We conclude that $\left(D_{k}^{h} v\right) \zeta \in W_{0}^{1,2}(B(z, \rho))$ with a norm independent of $h$. It now follows from a weak compactness argument that $v_{x_{k}} \zeta \in W_{0}^{1,2}(B(z, \rho))$ and

$$
\int_{B(z, \rho / 2)} \sum_{i=1}^{2}\left|v_{x_{k} x_{i}}\right|^{2} d x \leq \frac{c}{\rho^{2}} \int_{B(z, \rho)}\left|v_{x_{k}}-a\right|^{2} d x+\rho^{2}\left(\max _{B(0,1)} v\right)^{2} .
$$

This is what we wanted to prove.

Recall that if $\psi \in W^{1,2}(B(z, \rho))$ and $\psi_{B(z, \rho)}=\frac{1}{|B(z, \rho)|} \int_{B(z, \rho)} \psi d x$ then

$$
\left|\psi(x)-\psi_{B(z, \rho)}\right| \leq C \int_{B(z, \rho)} \frac{|\nabla \psi(y)|}{|x-y|} d y
$$


Let $\psi(x)=v_{x_{1}}(x)$ and $1 / 4>\rho$. It follows if $x \in B(0,1 / 4)$

$$
\begin{aligned}
\left|\psi(x)-\psi_{B(z, \rho)}\right| & \leq \int_{B(z, \rho)} \frac{|\nabla \psi(y)|}{|x-y|} d y \\
& =\int_{B(x, \delta)} \frac{|\nabla \psi(y)|}{|x-y|} d y+\int_{B(z, \rho) \backslash B(x, \delta)} \frac{|\nabla \psi(y)|}{|x-y|} d y
\end{aligned}
$$

and by Hölders inequality

$$
\begin{aligned}
& \int_{B(z, 2 \rho) \backslash B(x, \delta)} \frac{|\nabla \psi(y)|}{|x-y|} d y \\
& \quad \leq c\left(\int_{B(z, 2 \rho)}|\nabla \psi(y)|^{q} d y\right)^{1 / q}\left(\int_{B(z, 2 \rho) \backslash B(x, \delta)} \frac{1}{|x-y|^{\frac{q}{q-1}}} d y\right)^{\frac{q-1}{q}} \\
& \leq c\|\nabla \psi\|_{q} \delta^{(q-2) / q}
\end{aligned}
$$

and for the other integral we have the estimate

$$
\begin{aligned}
\int_{B(x, \delta)} \frac{|\nabla \psi(y)|}{|x-y|} d y & \leq \sum_{n=0}^{\infty} 2\left(\frac{2^{k}}{\delta}\right) \int_{\left\{2^{-(k+1)} \delta \leq|x-y| \leq 2^{-k} \delta\right\}}|\nabla \psi(y)| d y \\
& \leq 2 \sum_{k=0}^{\infty} \frac{\delta}{2^{k}} M(|\nabla \psi|) \leq 2 \delta M(|\nabla \psi|)
\end{aligned}
$$

Here $M(f)$ denotes the maximal function of $f$. We conclude

$$
\left|\psi(x)-\psi_{B(z, \rho)}\right| \leq 2 \delta M(|\nabla \psi|)+\delta^{(q-2) / q}\|\nabla \psi\|_{q}
$$

and if we choose

$$
\delta=\left(\frac{\|\nabla \psi\|_{q}}{2 M(|\nabla \psi|)}\right)^{q / 2}
$$

we get

$$
\left|\psi(x)-\psi_{B(z, \rho)}\right|^{2} \leq c M(|\nabla \psi|)^{2-q}\|\nabla \psi\|_{q}^{q}
$$

Integrating and applying Hölder's inequality yields for $1<q<\frac{3}{2}$

$$
\begin{aligned}
\left\|\psi(x)-\psi_{B(z, \rho)}\right\|_{2}^{2} & \leq \rho\left(\int_{B(z, 2 \rho)} M(|\nabla \psi|)^{4-2 q} d x\right)^{1 / 2}\|\nabla \psi\|_{q}^{q} \\
& \leq \rho\left(\int_{B(z, 2 \rho)}|\nabla \psi|^{4-2 q} d x\right)^{1 / 2}\|\nabla \psi\|_{q}^{q}
\end{aligned}
$$


If $B(z, \rho) \subset B(0,1 / 2)^{+}$Lemma 8 and (2.12) yield with $\psi=v_{x_{1}}, \psi_{B(z, \rho)}=a$

$$
\begin{aligned}
& \int_{B(z, \rho / 2)} \sum_{i}\left|v_{x_{1} x_{i}}\right|^{2} d x \\
& \leq \frac{c}{\rho}\left(\int_{B(z, \rho)}\left(\sum_{i, j}\left|v_{x_{j} x_{i}}\right|\right)^{4-2 q} d x\right)^{1 / 2} \int_{B(z, \rho)}\left(\sum_{i, j}\left|v_{x_{j} x_{i}}\right|\right)^{q} d x+c \rho^{2}\left(\max _{B(0,1)} v\right)^{2}
\end{aligned}
$$

If $B(z, \rho) \cap B(0,1 / 2)^{-} \neq \emptyset$ then we take $x=\left(x_{1}, x_{2}\right) \in B(z, \rho) \cap B(0,1 / 2)^{+}$ and let $x^{*}=\left(x_{1},-x_{2}\right)$. Note that if $x, y \in B(0,1)^{+}$then $|x-y| \leq\left|x^{*}-y\right|$. Since $\psi=0$ in $B(0,1)^{-}$we get

$$
\left|\psi_{B(z, \rho)}\right| \leq \int_{B(z, \rho)} \frac{|\nabla \psi(y)|}{\left|x^{*}-y\right|} d y \leq c \int_{B(z, \rho)} \frac{|\nabla \psi(y)|}{|x-y|} d y
$$

since $\psi\left(x^{*}\right)=0$. This allows us to get rid of $\psi_{B(z, \rho)}$ in our work above and we see that (2.13) holds in this case as well.

Lemma 9. Let $u$ be defined as above Lemma 7 and $v$ be defined as above Lemma 8. Then $v \in C^{4}\left(\overline{B(0,1 / 4)}^{+}\right)$and we have

$$
\left|D^{2} v\right|(x) \leq c \max _{B(0,1)} v
$$

for $x$ in $B(0,1 / 4)^{+}$. For the function $u$ we have $u \in C^{4}(\overline{\Omega \cap B(w, r / 8)})$ and

$$
\begin{aligned}
|\nabla u|(x) & \leq \frac{c}{r} \max _{B(w, r)} u \\
\left|D^{2} u\right|(x) & \leq \frac{c}{r^{2}} \max _{B(w, r)} u
\end{aligned}
$$

for $x \in B(w, r / 8) \cap \Omega$.

Proof. It follows from lemma 8 that $v$ is a strong solution of (2.7). Writing the equation in nondivergence form we obtain

$$
\left|v_{x_{2} x_{2}}\right|^{2} \leq c\left(\sum_{i=1}^{2}\left|v_{x_{1} x_{i}}\right|\right)^{2}+c\left(\max _{B(0,1)} v\right)^{2}
$$


Let $g=\sum\left|v_{x_{i} x_{j}}\right|$. We obtain

$$
\begin{aligned}
f_{B(z, \rho)} g^{2} d x & \leq f_{B(z, 2 \rho)} g^{q} d x\left(f_{B(z, 2 \rho)} g^{4-2 q} d x\right)^{1 / 2}+\rho^{2}\left(\max _{B(0,1)} v\right)^{2} \\
& \leq \epsilon\left(f_{B(z, 2 \rho)} g^{q} d x\right)^{2 / q}+C\left(f_{B(z, 2 \rho)} g^{4-2 q} d x\right)^{1 /(2-q)}+\rho^{2}\left(\max _{B(0,1)} v\right)^{2} \\
& \leq \epsilon f_{B(z, 2 \rho)} g^{2} d x+C\left(f_{B(z, 2 \rho)} g^{4-2 q} d x\right)^{1 /(2-q)}+\rho^{2}\left(\max _{B(0,1)} v\right)^{2}
\end{aligned}
$$

where we first used Youngs inequality and then Jensens inequality. In a ball $B(x, 2 t) \subset B(0,1)$ we define

$$
f(y)=\frac{\delta(y)}{2 t} g(y)
$$

where $\delta(y)$ is the distance from $y$ to $\partial B(x, 2 t)$ and note

$$
2 f(y) \geq g(y) \text { for } y \in B(x, t) \text { and } f(y) \leq g(y) \text { for } y \in B(x, 2 t)
$$

If $z \in B(x, 2 t)$ then

$$
\begin{aligned}
f_{B(z, \delta(z) / 2)} f^{2}(y) d y & \leq\left(\frac{2}{\delta(z)}\right)^{2} \int_{B(z, \delta(z) / 2)}\left(\frac{\delta(y)}{2 t}\right)^{2} g^{2}(y) d y \\
& \leq \frac{4}{t^{2}} \int_{B(x, 2 t)} g^{2}(y) d y=\lambda_{0}^{2}
\end{aligned}
$$

Let $\mu_{0}^{2}=\lambda_{0}^{2}+2 t^{2}\left(\max _{B(0,1)} v\right)^{2}$, take $\lambda \geq \lambda_{0}$, let $\mu^{2}=\lambda^{2}+2 t^{2}\left(\max _{B(0,1)} v\right)^{2}$ and $F(\mu)=\{z \in B(x, 2 t): f(z)>\mu\}$. Then it follows from differentiation theory that for almost every $z \in F(\mu)$ there exists $\rho>0$ such that

$$
f_{B(z, \rho)} f^{2} d x>\mu^{2}
$$

If $z \in F(\mu)$ and $\rho$ is sufficiently small it follows from (2.19) that we can select $\rho$ such that $10 \rho<\delta(z) / 2$ and

$$
\begin{aligned}
& f_{B(z, 10 \rho)} f^{2} d x<\mu^{2} \\
& f_{B(z, \rho)} f^{2} d x>\mu^{2}
\end{aligned}
$$


Then we obtain

$$
\begin{aligned}
\int_{B(z, 2 \rho)} g^{2} d y & \leq \int_{B(z, 10 \rho)} g^{2} d y \leq(10 \rho)^{2}\left(\frac{2 t}{\delta(z)}\right)^{2} f_{B(z, 10 \rho)} f^{2}(y) d y \\
& \leq(10 \rho)^{2}\left(\frac{2 t}{\delta(z)}\right)^{2} f_{B(z, \rho)} f^{2} d y \leq c \int_{B(z, \rho)} g^{2} d y
\end{aligned}
$$

Along with (2.17) this gives the estimate

$$
f_{B(z, \rho)} g^{2} d x \leq C\left(f_{B(z, 2 \rho)} g^{4-2 q} d x\right)^{1 /(2-q)}+2 t^{2}\left(\max _{B(0,1)} v\right)^{2}
$$

Since $10 \rho<\delta(z) / 2$ we have $\delta(z) / 4<\delta(y) / 2<\delta(z)$ for all $y \in B(z, 2 \rho)$. Therefore

$$
f_{B(z, \rho)} f^{2} d x \leq C\left(f_{B(z, 2 \rho)} f^{4-2 q} d x\right)^{1 /(2-q)}+2 t^{2}\left(\max _{B(0,1)} v\right)^{2}
$$

From (2.21) it now follows

$$
\lambda^{4-2 q} \leq C f_{B(z, 2 \rho)} f^{4-2 q} d x
$$

so

$$
\begin{aligned}
f_{B(z, 10 \rho)} f^{2} d x \leq \mu^{2} & =\lambda^{2}+2 t^{2}\left(\max _{B(0,1)} v\right)^{2} \\
& \leq C \lambda^{2 q-2}\left(f_{B(z, 2 \rho)} f^{4-2 q} d x\right)+2 t^{2}\left(\max _{B(0,1)} v\right)^{2}
\end{aligned}
$$

Let $E(\mu)=\{y \in B(x, 2 t): f(y)<\mu\}$ and note

$$
\int_{E(\delta \mu) \cap B(z, 2 \rho)} f^{4-2 q} d x \leq(\delta \mu)^{4-2 q} m(B(z, 2 \rho))
$$

where $m$ denotes two dimensional Lebesgue measure. By a well known covering theorem we can find a sequence of balls $\left\{B\left(z_{i}, \rho_{i}\right)\right\}$ such that $(2.21),(2.20)$ and (2.25) hold and

$$
\begin{gathered}
m\left(F(\mu) \backslash \bigcup_{i} B\left(z_{i}, 10 \rho_{i}\right)\right)=0 \\
B\left(z_{i}, 2 \rho_{i}\right) \cap B\left(z_{j}, 2 \rho_{j}\right)=\emptyset \quad i \neq j
\end{gathered}
$$


Now we have

$$
\begin{aligned}
\int_{F(\mu)} f^{2} d x & \leq \sum_{i} \int_{B\left(z_{i}, 10 \rho_{i}\right)} f^{2} d x \\
& \leq \lambda^{2 q-2}\left(\sum_{i} \int_{B\left(z_{i}, 2 \rho_{i}\right)} f^{4-2 q} d x\right)+2 t^{2}\left(\max _{B(0,1)} v\right)^{2} \\
& \leq C \lambda^{2 q-2}\left(\int_{F(\delta \mu)} f^{4-2 q} d x\right)+2 t^{2}\left(\max _{B(0,1)} v\right)^{2}
\end{aligned}
$$

Let $M$ be a large number and put

$$
\begin{gathered}
\tilde{f}=\min \{f, M\} \\
\tilde{F}(\mu)=\{z \in B(x, 2 t): \tilde{f}(z)>\mu\}
\end{gathered}
$$

Then it follows that

$$
\int_{\tilde{F}(\mu)} \tilde{f}^{2} d x \leq C \lambda^{2 q-2}\left(\int_{\tilde{F}(\delta \mu)} \tilde{f}^{4-2 q} d x\right)+2 t^{2}\left(\max _{B(0,1)} v\right)^{2}
$$

Now we get with integration by parts and Fubini's theorem

$$
\begin{aligned}
\int_{\tilde{F}\left(\mu_{0}\right)} \tilde{f}^{2+\gamma} d x= & \gamma \int_{\tilde{F}\left(\mu_{0}\right)} \tilde{f}^{2} \int_{0}^{\tilde{f}} \mu^{\gamma-1} d \mu d x=\gamma \int_{\mu_{0}}^{\infty} \mu^{\gamma-1} \int_{\tilde{F}(\mu)} \tilde{f}^{2} d x d \mu \\
\leq & \gamma \int_{\mu_{0}}^{\infty} \mu^{\gamma+2 q-3}\left(\int_{\tilde{F}(\delta \mu)} \tilde{f}^{4-2 q} d x\right)+2 t^{2}\left(\max _{B(0,1)} v\right)^{2} d \mu \\
= & \frac{(4-2 q) \gamma \delta^{4-2 q}}{\gamma+2 q-2} \int_{\mu_{0}}^{\infty} \mu^{1+\gamma} m(\tilde{F}(\delta \mu)) d \mu \\
& +\frac{\gamma}{\gamma+2 q-2} \mu_{0}^{\gamma+2 q-2}\left(\int_{\tilde{F}\left(\delta \mu_{0}\right)} \tilde{f}^{4-2 q} d x\right)+2 t^{2}\left(\max _{B(0,1)} v\right)^{2}
\end{aligned}
$$

By choosing $\delta$ small enough this gives

$$
\int_{\tilde{F}\left(\mu_{0}\right)} \tilde{f}^{2+\gamma} d x \leq C \mu_{0}^{\gamma+2 q-2}\left(\int_{B(x, 2 t)} \tilde{f}^{4-2 q} d x\right)+t^{2}\left(\max _{B(0,1)} v\right)^{2}
$$

By the monotone convergence theorem we see that this inequality holds for $f$ and by (2.18), (2.19) and Jensen's inequality that

$$
\left(f_{B(x, t)} g^{2+\gamma} d x\right)^{1 /(2+\gamma)} \leq C\left(f_{B(x, 2 t)} g^{2} d x\right)^{1 / 2}+c\left(\max _{B(0,1)} v\right)^{2}
$$


This implies that $v \in W^{2,2+\gamma}\left(B(0,1 / 4)^{+}\right)$and from Morrey's inequality we see that $v \in C^{1, \alpha}\left(\overline{B(0,1 / 4)}^{+}\right)$and the $C^{1, \alpha}$ norm of $v$ is bounded by the $W^{2,2+\gamma}$ norm of $v$. If we write (2.7) in nondivergence form we obtain an equation

$$
\sum_{i, j} a_{i j}(x, \nabla v) v_{x_{i} x_{j}}+b(x, \nabla v)=0
$$

Since the matrix $A$ in $(2.7)$ is smooth and the function $v \in C^{1, \alpha}\left(\overline{B(0,1 / 4)}^{+}\right)$ it follows that $a_{i j} \in C^{\alpha}\left(\overline{B(0,1 / 4)}^{+}\right)$. Also (2.8) gives us that the equation is strictly elliptic. Then lemma 9 follows from boundary Schauder estimates (see $[18$, chapter 6]).

\section{Preliminary reductions}

Assume $\Omega$ is a bounded domain of class $C^{4}$. This means that for each $y \in \partial \Omega$ there exists $s>0$ such that $B(y, s) \cap \partial \Omega$ is a part of the graph of a four times continuously differentiable function defined on a line in $\mathbb{R}^{2}$ and $B(y, s) \cap \Omega$ lies above the graph. We use compactness and a standard covering argument to obtain $y^{1}, \ldots, y^{N} \in \partial \Omega$ such that

$$
\partial \Omega \subset \bigcup_{i=1}^{N} B\left(y^{i}, 100 r\right) \text { and } B\left(y^{i}, 10 r\right) \cap B\left(y^{j}, 10 r\right)=\emptyset, \quad i \neq j
$$

If $r$ is sufficiently small and $y=y^{i}$ then it follows from the implicit function theorem that there exists a function $\theta=\theta(\cdot, y)$ four times continuously differentiable on $\mathbb{R}$ with $\theta(0)=0$ and $\theta_{x}(0)=0$ such that after a rotation of the axes, if necessary:

$$
\begin{aligned}
\partial \Omega \cap B\left(y, 1000 r^{1 / 2}\right) & \subset\left\{\left(x_{1}+y_{1}, \theta\left(x_{1}\right)+y_{2}\right): x_{1} \in \mathbb{R}\right\} \\
\Omega \cap B\left(y, 1000 r^{1 / 2}\right) & \subset\left\{\left(x_{1}+y_{1}, x_{2}\right): x_{2}-y_{2}>\theta\left(x_{1}\right), x_{1} \in \mathbb{R}\right\}
\end{aligned}
$$

Let

$$
K_{1}=\max _{y \in\left\{y^{i}\right\}_{1}^{N}}\left(\max _{x \in \partial \Omega \cap B\left(y, 100 r^{1 / 2}\right)} \sum_{k=1}^{4}\left|\theta^{(k)}(\cdot, y)\right|\right)
$$

and for $0<\epsilon<\sigma_{0} \leq 10^{-3}$ choose $r_{0}>0$ so small that for $0<r \leq r_{0}$

$$
K_{1} r^{1 / 2} \leq 10^{-3} r^{1 / 4} \leq 10^{-9} \epsilon^{4}
$$

which is possible since $K_{1}<+\infty$ by compactness of $\partial \Omega$. Let $u$ be a function satisfying (1.5a)-(1.5b) with $D$ replaced by $\Omega$ and assume that $u \in C^{4}(\bar{\Omega})$ 
and $|\nabla u|>1$ on $\partial \Omega$. Let

$$
K_{2}=\max _{y \in\left\{y^{i}\right\}_{1}^{N}}\left(\max _{x \in \bar{\Omega} \cap B\left(y, 100 r^{1 / 2}\right)} \sum\left|\partial_{\alpha} u(x)\right|\right)
$$

where $\alpha=\left(\alpha_{1}, \alpha_{2}\right)$ is a multiindex and $0 \leq|\alpha| \leq 4$. Choose $r_{0}$ even smaller so that if $0<r \leq r_{0}$ then

$$
K_{2} r^{1 / 2} \leq 10^{-3} r^{1 / 4} \leq 10^{-9} \epsilon^{4}
$$

Let $l$ be the largest nonnegative integer such that $2^{-l} \sigma_{0}>\epsilon$ and let $\sigma_{k}=$ $2^{-k} \sigma_{0}$ for $k=0,1, \ldots$. Put

$$
E_{k}=\left\{x \in \partial \Omega: 1+\sigma_{k}<|\nabla u(x)| \leq 1+\sigma_{k-1}\right\},
$$

for $1 \leq k \leq l+1$ and

$$
E_{0}=\left\{x \in \partial \Omega:|\nabla u(x)|>1+\sigma_{0}\right\}
$$

Let $\psi \geq 0$ be a $C^{\infty}$ function on $\mathbb{R}$ with $\max \psi=1$ and support in the unit interval. Let $L$ be the set of all $y \in\left\{y^{i}\right\}_{1}^{N}$ for which

$$
B(y, 100 r) \cap \bigcup_{k=0}^{l+1} E_{k} \neq \emptyset
$$

For a fixed $y=\left(y_{1}, y_{2}\right) \in L$ let $j$ be the smallest nonnegative integer with

$$
B(y, 100 r) \cap E_{j} \neq \emptyset
$$

Put

$$
\xi\left(x_{1}\right)=\theta\left(x_{1}\right)-\sigma_{j}^{4} r \psi\left(x_{1} / r \sigma_{j}^{2}\right)+y_{2} \quad x_{1} \in \mathbb{R}
$$

Now we define $\Omega^{\prime}$ as follows

$$
\begin{array}{ll}
\text { (i) } & \Omega \backslash \bigcup_{y \in L} B(y, 10 r)=\Omega^{\prime} \backslash \bigcup_{y \in L} B(y, 10 r) \\
\text { (ii) } & \partial \Omega^{\prime} \cap B(y, 10 r)=\left\{\left(x_{1}+y_{1}, \xi\left(x_{1}\right)\right): x_{1} \in \mathbb{R}\right\} \cap B(y, 10 r) \\
\text { (iii) } & \left.\Omega^{\prime} \cap B(y, 10 r)=\left\{\left(x_{1}+y_{1}, x_{2}\right): x_{2}>\xi\left(x_{1}\right)\right\}\right\} \cap B(y, 10 r) .
\end{array}
$$

Clearly $\Omega^{\prime}$ is of class $C^{4}$.

Lemma 10. Let $u^{\prime}$ be defined by $(1.5 \mathrm{a})-(1.5 \mathrm{~b})$ with $D$ replaced by $\Omega^{\prime}$. Then $u^{\prime} \in C^{4}\left(\bar{\Omega}^{\prime}\right)$ and if $r_{0}$ is small enough

$$
\left|\nabla u^{\prime}(x)\right|>1, \quad x \in \partial \Omega^{\prime} .
$$


Proof. First $u^{\prime} \in C^{4}(\bar{\Omega})$ follows from lemma 9 since $\nabla u^{\prime} \neq 0$ in $\Omega^{\prime}$ (see [15]). If $x \in \partial \Omega^{\prime} \cap \partial \Omega$ then it follows from the maximum principle that (3.6) is true. Let $Z(y, t)=\left\{\left(x_{1}, x_{2}\right):\left|x_{i}-y_{i}\right|<t, i=1,2\right\}$. If $x \in \partial \Omega^{\prime} \backslash \partial \Omega$ we first note that since $\psi$ has support in the unit interval

$$
\left(\partial \Omega^{\prime} \backslash \partial \Omega\right) \cap B(y, 10 r) \subset Z(y, r)
$$

whenever $y \in L$. From the maximum principle and (3.7) it follows that to prove (3.6) it suffices to show that

$$
\left|\nabla u^{*}(x)\right|>1 \quad x \in Z(y, r) \cap \partial \Omega^{*}
$$

where $\Omega^{*}$ is obtained by adding just one bump to $\Omega$ at the point $y$ and $u^{*}$ satisfies (1.5a)-(1.5b) with $D$ replaced by $\Omega^{*}$.

We note that since $|\nabla u(x)|>1$ on $\partial \Omega$ it follows from (3.2) that $u_{x_{2}}>1 / 2$ when $x \in Z(y, r)$. Let $t_{0}=\min _{Y} u$ where $Y=\left\{\left(x_{1}, x_{2}\right) \in \partial Z(y, r) \cap \Omega\right.$ : $\left.\left|y_{2}-x_{2}\right|=r\right\}$. Note that $c t_{0} \geq \max _{\Omega \cap Z(y, r)} u$ by Harnack's inequality. Let $U=\Omega \cap Z(y, r) \cap\left\{u<t_{0}\right\}$ and note that $u$ is increasing on $\partial U \cap \partial Z(y, r)$. Let $U^{*}=\Omega^{*} \cap Z(y, r) \cap\left\{u(x)<t_{0}\right\}$. Define $v$ to be the $p$ harmonic function in $U^{*}$ such that $v=0$ on $\partial \Omega^{*}$ and $v=u$ on $\partial U^{*} \backslash \partial \Omega^{*}$. Note that $v \leq u^{*}$ in $U^{*}$ by the boundary maximum principle so it suffices to show $|\nabla v|>1$ on $\partial \Omega^{*}$. In order to do this we need to apply the estimates in section 2 to the function $v$. This requires us to show that $\nabla v \neq 0$.

Consider the function $v^{\epsilon}$ in $U^{*}$ which solves the equation

$$
\nabla \cdot\left(\left(\left|\nabla v^{\epsilon}\right|^{2}+\epsilon\right)^{p / 2-1} \nabla v^{\epsilon}\right)
$$

and satisfies $v^{\epsilon}=v$ on $\partial U^{*}$. This equation is strictly elliptic so it follows from Schauder estimates (see [13] or [18]) that $v^{\epsilon}$ is real analytic in the interior of $U^{*}$ and continous in the closure of $U^{*}$ (see [13]). If $t<t_{0}$ the set $\partial U \cap\{u=t\}$ contains exactly two points. Since $v^{\epsilon}=u$ on $\partial U^{*} \backslash \partial \Omega^{*}$ the set $\left\{v^{\epsilon}(x)>s\right\}$ is connected in $U^{*}\left(s<t_{0}\right)$ since each component must intersect the boundary of $U$ by the maximum principle for $v^{\epsilon}$. We note that it follows from [15] that if $\left|\nabla v^{\epsilon}\left(x_{0}\right)\right|=0$ then $\left\{v^{\epsilon}(x)>v^{\epsilon}\left(x_{0}\right)\right\}$ can not be connected. Since we have already concluded that these sets are connected we see that $\nabla v^{\epsilon} \neq 0$ in $U^{*}$. Now one can argue as in [15] to obtain $\nabla v \neq 0$ in $U^{*}$. Since $u$ is Hölder continous there exists a $\lambda$ which depends only on $p$ so that $u<t_{0}$ in $Z(y, \lambda r)$ so $Z(y, \lambda r) \cap \Omega^{*} \subset U^{*}$. Thus we have $\nabla v \neq 0$ in $Z(y, \lambda r)$.

Now we can apply lemma 9 to $v$ and obtain

$$
\max _{Z(y, \lambda r / 8) \cap \Omega^{*}}\left|D^{2} v\right| \leq \frac{c}{r^{2}} \max _{Z(y, \lambda r)} v \leq \frac{c}{r^{2}} \max _{Z(y, r)} u \leq \frac{c}{r}|\nabla u|(t)
$$

for $t \in Z(y, r)$. 
Let $\sigma_{0}$ be so small that $\sigma_{0}<\lambda / 8$. By the maximum principle $|\nabla v| \geq|\nabla u|$ on $\partial \Omega \cap \partial \Omega^{*}$ and from our construction we know that there exists some point $x \in \partial \Omega \cap B(y, 100 r)$ such that $1+\sigma_{j} \leq|\nabla u|(x)$. From (3.2) it follows that $|\nabla u|(x) \geq 1+\sigma_{j} / 2$ for all $x \in \partial \Omega \cap B(y, 100 r)$. Pick a point $z \in \partial \Omega \cap \partial \Omega^{*} \cap B\left(y, \sigma_{j}^{2} r\right)$. By (3.2) we see that

$$
|\nabla u(t)| \leq|\nabla u(z)|+10^{-9} \epsilon^{4} r^{1 / 2}
$$

for $t \in Z(y, r)$. Choosing $\sigma_{0}$ smaller so that $C \sigma_{0}<10^{-3}$ and using the mean value theorem and (3.2) we obtain for $x \in \partial \Omega^{*} \cap B\left(y, \sigma_{j}^{2} r\right)$

$$
\begin{aligned}
|\nabla v(z)-\nabla v(x)| & \leq \max _{Z(y, r) \cap \Omega^{*}}\left|D^{2} v\right||z-x| \\
& \leq c|\nabla u|(t) \frac{|z-x|}{r} \leq 10^{-3} \sigma_{j}|\nabla u|(z)+10^{-12} \epsilon^{4} r^{1 / 2} \sigma_{j}
\end{aligned}
$$

and since $|\nabla v|(z) \geq|\nabla u|(z)$

$$
\begin{aligned}
|\nabla v|(x) & \geq\left(1-10^{-3} \sigma_{j}\right)|\nabla v|(z)-10^{-12} \epsilon^{4} r^{1 / 2} \sigma_{j} \\
& \geq\left(1-10^{-3} \sigma_{j}\right)\left(1+\frac{1}{2} \sigma_{j}\right)-10^{-12} \epsilon^{4} r^{1 / 2} \sigma_{j}>1 .
\end{aligned}
$$

Which is what we needed to prove.

Lemma 11. Let $\Omega, \Omega^{\prime}$ be as above. If $\epsilon \leq t \leq 1$

$$
H^{1}\left(\partial \Omega^{\prime}\right) \geq H^{1}(\partial \Omega)+\eta(t) H^{1}\{x:|\nabla u(x)|>1+t\}
$$

if $p<2$ but

$$
H^{1}\left(\partial \Omega^{\prime}\right) \geq H^{1}(\partial \Omega)+\eta(t) H^{1}\{x:|\nabla u(x)|<1-t\}
$$

if $p>2$. Here $\eta$ is a positive function on $] 0, \infty[$.

Proof. To prove (3.12) let

$$
c_{2}=\int_{\mathbb{R}}\left|\psi^{\prime}(x)\right|^{2} d x
$$

and choose $\sigma_{0}$ even smaller so that

$$
\sigma_{0} \leq c_{2} \leq 2\left(\max _{\mathbb{R}}\left|\psi^{\prime}\right|\right)^{2} \leq \sigma_{0}^{-1} 10^{-6}
$$


Then it follows from (3.1) and the definition of $\sigma_{j}$

$$
\begin{aligned}
H^{1}(Z(y, r) & \left.\cap \partial \Omega^{\prime}\right)=\int_{-r}^{r} \sqrt{1+\left|\xi^{\prime}\right|^{2}} d x \\
& \geq \int_{-r}^{r} \sqrt{1+\sigma_{j}^{4}\left|\psi^{\prime}(x / r)\right|^{2}} d x-2 \epsilon^{8} r \\
& =r \int_{-1}^{1} \sqrt{1+\sigma_{j}^{4}\left|\psi^{\prime}(x)\right|^{2}} d x-2 \epsilon^{8} r \\
& \geq\left(1+\frac{1}{4} \sigma_{j}^{4} c_{2}-\epsilon^{8}\right) 2 r \geq \frac{1}{8} \sigma_{j}^{4} c_{2} 2 r+H^{1}(Z(y, r) \cap \partial \Omega) .
\end{aligned}
$$

Take $t \geq \epsilon$ and let $k$ be the least nonnegative integer such that $t \geq \sigma_{k}$, $0 \leq k \leq l+1$. Let $J=J(k)$ be the set of all $i$ such that (3.5) holds with $y=y^{i}$ and $j \leq k$. From (3.1) it is clear that

$$
\begin{aligned}
H^{1}\{x \in \partial \Omega:|\nabla u(x)| \geq 1+t\} & \leq H^{1}\left(\bigcup_{i \in J} B\left(y^{i}, 100 r\right) \cap \partial \Omega\right) \\
& \leq 2 \sum_{i \in J} 200 r
\end{aligned}
$$

and we conclude that

$$
H^{1}\left(\partial \Omega^{\prime}\right) \geq H^{1}(\partial \Omega)+c_{3} \sigma_{k}^{4} H^{1}\{x \in \partial \Omega: \mid \nabla u(x)>1+t\}
$$

Let

$$
\eta(t)= \begin{cases}c_{3} \sigma_{0}^{4} & \text { if } \sigma_{0} \leq t \\ c_{3} \sigma_{k}^{4} & \text { if } \sigma_{k} \leq t<\sigma_{k-1}, k=1,2, \ldots\end{cases}
$$

Since $\eta$ does not depend on $\Omega$ this proves (3.12). The case when $p>2$ is similar.

\section{Proof of Theorem 1}

Lemma 12. Let $u, \Omega$ be as above. If $1<p<2$ then

$$
\int_{\partial \Omega}|\nabla u|^{p-1} \log |\nabla u| d H^{1} \leq C
$$

and if $p>2$ then

$$
\int_{\partial \Omega}|\nabla u|^{p-1} \log |\nabla u| d H^{1} \geq C
$$

where the constant $C$ depends only on $F$. 
Proof. We proceed as in [2]. Note that if $\eta \in \mathbb{R}^{2}$ and $|\eta|=1$ then $\zeta=\langle\nabla u, \eta\rangle$ is a strong solution to

$$
L \zeta=\nabla \cdot\left((p-2)|\nabla u|^{p-4}\langle\nabla u, \nabla \zeta\rangle \nabla u+|\nabla u|^{p-2} \nabla \zeta\right)=0
$$

in $\Omega \cap N$ since $\nabla u \neq 0$. In other words

$$
L \zeta=\sum_{i, k=1}^{2} \frac{\partial}{\partial x_{i}}\left(a_{i k}(x) \zeta_{x_{k}}(x)\right)=0
$$

where

$$
a_{i k}(x)=|\nabla u|^{p-4}\left((p-2) u_{x_{i}} u_{x_{k}}+\delta_{i k}|\nabla u|^{2}\right)(x)
$$

and $\delta_{i j}$ is the Kronecker $\delta$. Note that

$$
L u=(p-1) \nabla \cdot\left(|\nabla u|^{p-2} \nabla u\right)=0
$$

Since the equation is rotationally invariant we can assume that $\nabla u(x)=$ $(|\nabla u(x)|, 0)$. Let $v=\log |\nabla u(x)|$. Then

$$
v_{x_{k}}=|\nabla u|^{-2} \sum_{l=1}^{2} u_{x_{l}} u_{x_{l} x_{k}}
$$

and so

$$
L v=\sum_{i, k=1}^{2} \frac{\partial\left(a_{i k} v_{x_{k}}\right)}{\partial x_{i}}=\sum_{i=1}^{2} \frac{\partial}{\partial x_{i}}\left(|\nabla u|^{-2} \sum_{k, l=1}^{2} a_{i k} u_{x_{l}} u_{x_{l} x_{k}}\right) .
$$

Using (4.3) on the righthand side we get

$$
\begin{aligned}
L v= & -2|\nabla u|^{-4} \sum_{i, k, l, m=1}^{2} a_{i k}\left(u_{x_{l}} u_{x_{l} x_{k}} u_{x_{m}} u_{x_{m} x_{i}}\right) \\
& +|\nabla u|^{-2} \sum_{i, k, l=1}^{2} a_{i k} u_{x_{l} x_{i}} u_{x_{l} x_{k}}=T_{1}+T_{2} .
\end{aligned}
$$

From the definiton of the $a_{i k}$ 's and our assumption that $\nabla u(x)=(|\nabla u(x)|, 0)$ we see at $x$

$$
a_{11}=(p-1)|\nabla u|^{p-2}, a_{22}=|\nabla u|^{p-2} \text { and } a_{12}=a_{21}=0
$$

and also from (4.5)

$$
(p-1) u_{x_{1} x_{1}}+u_{x_{2} x_{2}}=0
$$


Using this in the definitions of $T_{1}, T_{2}$ we obtain at $x$

$$
T_{1}=-2|\nabla u|^{p-4}\left((p-1)\left(u_{x_{1} x_{1}}\right)^{2}+\left(u_{x_{1} x_{2}}\right)^{2}\right)
$$

and

$$
T_{2}=p|\nabla u|^{p-4}\left((p-1)\left(u_{x_{1} x_{1}}\right)^{2}+\left(u_{x_{1} x_{2}}\right)^{2}\right)
$$

and we conclude

$$
L v=(p-2)|\nabla u|^{p-4}\left((p-1)\left(u_{x_{1} x_{1}}\right)^{2}+\left(u_{x_{1} x_{2}}\right)^{2}\right)
$$

so $L v \leq 0$ when $1<p<2$ and $L v \geq 0$ when $p>2$. Since $u$ is smooth and $\nabla u \neq 0$ and $\partial \Omega$ is smooth we can apply the divergence theorem to the vector field whose $i$ th component is

$$
u \sum_{k=1}^{2} a_{i k} v_{x_{k}}-v \sum_{k=1}^{2} a_{i k} u_{x_{k}}
$$

in the region $\Omega \backslash G$ where $G$ is a region with smooth boundary which contains the set $F$ in its interior. If $1<p<2$ we obtain

$$
\begin{aligned}
0 & \geq \int_{\Omega \backslash G} u\left(\sum_{k=1}^{2} \frac{\partial}{\partial x_{i}}\left(a_{i k} v_{x_{k}}\right)\right)-v\left(\sum_{k=1}^{2} \frac{\partial}{\partial x_{i}}\left(a_{i k} u_{x_{k}}\right)\right) d x \\
& =\int_{\partial \Omega}|\nabla u|^{p-1} \log |\nabla u| d H^{1}+\int_{\partial G} \sum_{i=1}^{2}\left(u \sum_{k=1}^{2} a_{i k} v_{x_{k}}-v \sum_{k=1}^{2} a_{i k} u_{x_{k}}\right) \eta_{i} d H^{1}
\end{aligned}
$$

where $\eta$ is the outward unit normal for $\Omega \backslash G$ on $\partial G$ and we used the fact that $u=0$ on $\partial \Omega$ and $\eta=-\frac{\nabla u}{|\nabla u|}$ on $\partial \Omega$. This gives (1.9) and (1.10) where the constant is determined by the integral over $\partial G$ which is independent of $\Omega$.

Remember that $\psi$ is a $C^{\infty}$ function on $\mathbb{R}$ with $\max \psi=1$ and support in the unit interval. Also, in section $3 \sigma_{0}, 0<\sigma_{0} \leq 10^{-3}$ was chosen so that (3.14) was true. Finally, for a given $\epsilon, 0<\epsilon \leq \sigma_{0} r_{0}$ was chosen so small that the estimates in section 3 are true for $0<r \leq r_{0}$. We describe the construction of $D$ in more detail. We only describe the case of "pushing out" since the other case is similar. Let $D_{0}$ be a domain such that $F \subset D_{0}$ and the function $u_{0}$ which satisfies (1.5a)-(1.5b) for $D_{0}$ also satisfies $\left|\nabla u_{0}\right|>1$ on $\partial D_{0}$. Let $\rho=d(\partial \Omega, F)$. Let $\epsilon_{0}=\sigma_{0}$ and $\epsilon_{k}=2^{-k} \epsilon_{0}$ for $k=1,2, \ldots$ Choose a covering $L_{1}=\left\{B\left(z_{0}^{i}, t_{0}^{i}\right)\right\}, 1 \leq i \leq k_{0}$ of $\partial D_{0}$ such that $t_{0}^{i} \leq 1 / 2$ for all $i$ and

$$
2 \sum_{i=1}^{k_{0}} t_{0}^{i} \leq H^{1}\left(\partial D_{0}\right)+\frac{1}{2}
$$


Since $D_{0}$ is compact we can assume $k_{0}<\infty$. Let $2 r_{1}^{\prime}>0$ be the distance from $\partial D_{0}$ to $\mathbb{R}^{2} \backslash \bigcup_{1}^{k_{0}} B\left(z_{0}^{i}, t_{0}^{i}\right)$. Set $\Omega=D_{0}, \epsilon=\epsilon_{1}$ and $r_{1}=$ $\min \left\{r_{1}^{\prime}, r_{0}\left(\epsilon_{1}, K_{1}, K_{2}\right), 10^{-9} \rho\right\}$ where $K_{1}$ and $K_{2}$ are defined relative to $D_{0}$, $u_{0}$ as in section 3 . Then we do as in section 3 to obtain $D_{1}=\Omega^{\prime}$. Now suppose for some $m \geq 1$ we have defined $\left\{D_{k}\right\}_{0}^{m},\left\{L_{k}\right\}_{0}^{m},\left\{r_{k}^{\prime}\right\}_{0}^{m}$ and $\left\{r_{k}\right\}_{0}^{m}$. Let $L_{m+1}=\left\{B\left(z_{m}^{i}, t_{m}^{i}\right)\right\}_{1}^{k_{m}}$ be a covering of $\partial D_{m}$ such that $t_{m}^{i} \leq 2^{-(m+1)}$, $1 \leq i \leq k_{m}$ and

$$
2 \sum_{i=1}^{k_{m}} t_{m}^{i} \leq H^{1}\left(\partial D_{m}\right)+2^{-(m+1)}
$$

Let $2 r_{m}^{\prime}>0$ be the distance between $\partial D_{m}$ and $\mathbb{R}^{2} \backslash \bigcup_{1}^{k_{m}} B\left(z_{m}^{i}, t_{m}^{i}\right)$. Let $\Omega=D_{m}, \epsilon=\epsilon_{m}$ and $r=r_{m+1}=\min \left\{r_{m}^{\prime}, r_{0}\left(\epsilon_{m+1}, K_{1}, K_{2}\right), 10^{-4 m} r_{m}\right\}$ where $K_{1}$ and $K_{2}$ are defined relative to $D_{m}, u_{m}$ as in Section 3. Then we do as in Section 3 to obtain $D_{m+1}=\Omega^{\prime} \supset D_{m}$. By induction we get $\left\{D_{k}\right\}_{0}^{\infty},\left\{L_{k}\right\}_{1}^{\infty}$, $\left\{r_{k}^{\prime}\right\}_{1}^{\infty}$ and $\left\{r_{k}\right\}_{1}^{\infty}$. Finally define $D$ to be the union of the sets $D_{k}$

Lemma 13. Let $D, D_{k}, k=1,2, \ldots$ be as above. Then $D$ is a quasicircle which is not convex. For $D_{k}$ we have $\mu_{k}\left(\partial D_{k}\right) \leq C$ where $C$ is inedependent of $k$ and $\mu_{k}$ is the measure corresponding to $u_{k}$ as in (1.2).

Proof. To prove that $D$ is a quasicircle it suffices to show that $\partial D_{m}$ satisfies the Ahlfors three point condition for $m=1,2, \ldots$ with constant independent of $m$. Once we have proved this we get a sequence $\left\{f_{m}\right\}$ of quaisconformal mappings of $\mathbb{R}^{2}$ with

$$
f_{m}(\partial B(0,1))=\partial D_{m} \text { and }\left|\left(f_{m}\right)_{\bar{z}}\right| \leq k\left|\left(f_{m}\right)_{z}\right|
$$

where $0<k<1$ is independent of $m$. Since a subsequence of $\left\{f_{m}\right\}$ converges uniformly on compact subsets of $\mathbb{R}^{2}$ to a quasiconformal $f: \mathbb{R}^{2} \rightarrow \mathbb{R}^{2}$ we see that (4.12) holds with $f_{m}, D_{m}$ replaced by $f, D$. To show that $\partial D_{m}$ satisfies the Ahlfors three point condition independent of $m$ we first find a constant $C$ such that $\left|z_{1}-z_{3}\right|<C\left|z_{1}-z_{2}\right|$ for $z_{1}, z_{2}, z_{3}$ on the graph of $\psi$ and $z_{3}$ between $z_{1}$ and $z_{2}$. Now suppose $z_{1}, z_{2}, z_{3}$ lie on $\partial D_{m}$ and $\left|z_{1}-z_{2}\right|<10 r_{m}$. Let $\xi$ be a function whose graph is after a rotation and translation $\partial D_{m} \cap B\left(z_{1}, 10 r_{m}\right)$. By (3.1) the distance $|\xi(x)-\psi(x)|$ is less than $10^{-9} \epsilon_{m}^{4}|x|$ which implies that the graph of $\xi$ and therefore $\partial D_{m} \cap B\left(z_{1}, 10 r_{m}\right)$ satisfies the Ahlfors three point condition with a slightly larger constant $C$ but still independent of $m$. If $\left|z_{1}-z_{2}\right|>10 r_{m}$ we find $k<m$ such that $\left|z_{1}-z_{2}\right|<10 r_{k}$ but $\left|z_{1}-z_{2}\right|>100 r_{k+1}$. Let $z^{*}$ be the projection of $z \in \partial D_{m}$ on $\partial D_{k}$. Then $\left|z_{1}-z_{2}\right|>\left|z_{1}^{*}-z_{2}^{*}\right|-\eta r_{k}$ where $\eta$ is small and likewise $\left|z_{1}-z_{3}\right|<\left|z_{1}^{*}-z_{3}^{*}\right|+\eta r_{k}$. From this it follows that $\left|z_{1}-z_{3}\right|<2 C\left|z_{1}-z_{3}\right|$ for all $m$. 
To prove that $\mu_{k}\left(\partial D_{k}\right) \leq C$ where $C$ is independent of $k$ we recall that $\mu(B(x, r)) \leq c r^{2-p}\left(\max _{B(x, 2 r)} u\right)^{p-1}$ for any measure defined by (1.2). This estimate is proved in [5] and our claim follows immediately by covering the boundaries of the domains $D_{k}$ with balls and then applying the estimate in each ball since $u_{k}(x) \leq 1$ for all $x \in D_{k}$. To see that the domain is not convex note that the function $\psi$ can be chosen so that $D_{m}$ has the property that there exist points $x, y \in D_{m}$ such that

$$
\max _{t \in[0,1]} d\left(t x+(t-1) y, D_{m}\right)>\frac{\epsilon_{m}^{4} r_{m}}{8}>5^{4 m} r_{m+1} \frac{\epsilon_{0}}{8}>r_{m+1}
$$

if $m$ is large enough. It is clear from the construction described above that if $z \in D$ then $d\left(z, D_{m}\right)<r_{m+1}$ so the line segment between $x$ and $y$ does not lie in $D$. However $x, y \in D$ so $D$ is not convex.

The proof of Theorem 1 follows from the above lemmas and the argument at the end of section 1 once we prove (1.14). The proof that $\left.\left.H^{1}\right|_{\partial D_{m}} \rightarrow H^{1}\right|_{\partial D}$ in [16] applies to our case without change. For completeness we give a brief outline. First show that there exists a mapping $h_{m}$ from $\partial D_{m}$ to $\partial D_{m+1}$ which satisfies

$$
\left|h_{m}(x)-h_{m}(z)\right| \geq\left(1-c r_{m}^{1 / 2}\right)|x-z|
$$

Then let

$$
p_{j}(x)=\lim _{k \rightarrow \infty} h_{k} \circ \cdots \circ h_{j+1}(x) \text { for } x \in \partial D_{j} .
$$

If

$$
e_{j}=\prod_{m=j+1}^{\infty}\left(1-c r_{m}^{1 / 2}\right)
$$

it follows that

$$
e_{j}|x-y| \leq\left|p_{j}(x)-p_{j}(y)\right|, \quad x, y \in \partial D_{j},
$$

and if $q_{j}$ is the inverse of $p_{j}$ we have

$$
\left|q_{j}(x)-q_{j}(y)\right| \leq e_{j}^{-1}|x-y|
$$

when $x, y \in \partial D$. Next we use Kirsbraun's Theorem (see [7]) to obtain an extension of $q_{j}$ to $\mathbb{R}^{2}$ such that (4.13) holds whenever $x, y \in \mathbb{R}^{2}$. Let $\nu(E)=H^{1}\left(q_{j}^{-1}(E) \cap \partial D\right)$. Then we have

$$
H^{1}\left(E \cap \partial D_{j}\right) \leq e_{j} \nu(E)
$$

Also note that it follows from the definition of the $r_{m}$ 's that $e_{j} \rightarrow 1$ when $j \rightarrow \infty$. Let $g \geq 0$ be a continuous function. Then it follows from the 
change of variables formula that

$$
e_{j} \int_{\partial D_{j}} g d H^{1} \leq \int_{\mathbb{R}^{n}} g d \nu=\int_{\partial D} g \circ q_{j} d H^{1}
$$

If we let $j \rightarrow \infty$ then $q_{j}(x) \rightarrow x$ uniformly on compact subsets of $\mathbb{R}^{n}$ so

$$
\int_{\partial D} g \circ q_{j} d H^{1} \rightarrow \int_{\partial D} g d H^{1}
$$

Hence from (4.14) we have

$$
\limsup _{k \rightarrow \infty} \int_{\partial D_{n}} g d H^{1} \leq \int_{\partial D} g d H^{1}
$$

From our construction of $D$ it follows that

$$
H^{1}(\partial D) \leq \liminf _{m \rightarrow \infty} H^{1}\left(\partial D_{m}\right)
$$

If $0 \leq g \leq 1$ then it follows that

$$
\begin{aligned}
H^{1}(\partial D) & \leq \liminf _{k \rightarrow \infty} H^{1}\left(\partial D_{n_{k}}\right) \\
& \leq \liminf _{k \rightarrow \infty} \int_{\partial D_{k}} g d H^{1}+\limsup _{k \rightarrow \infty} \int_{\partial D_{k}}(1-g) d H^{1} \\
& \leq \limsup _{k \rightarrow \infty} \int_{\partial D_{n}} g d H^{1}+\limsup _{k \rightarrow \infty} \int_{\partial D_{n}}(1-g) d H^{1} \\
& \leq \int_{\partial D} g d H^{1}+\int_{\partial D}(1-g) d H^{1}=H^{1}(\partial D)
\end{aligned}
$$

Thus equality holds everywhere so

$$
\lim _{k \rightarrow \infty} \int_{\partial D_{n}} g d H^{1}=\int_{\partial D} g d H^{1}
$$

which is what we wanted to prove.

To show that $\mu_{k} \rightarrow \mu$ we note that if we are pushing out then $u(x)<\epsilon$ on $\partial D_{n}$ for $n$ large enough. Therefore $u(x)<u_{n}(x)+\epsilon$ in $D_{n}$ in other words $u(x)-u_{n}(x)<\epsilon$ in $D_{n}$. Elsewhere $u_{n}(x)=0$ and $u(x)<\epsilon$ so $u_{n} \rightarrow u$ uniformly. Since the measures $\mu_{n}$ are bounded we have a subsequence which is weakly convergent to some measure $\nu$. Now

$$
\begin{aligned}
\int \phi d \nu & =\lim _{n \rightarrow \infty} \int \phi d \mu_{n}=\lim _{n \rightarrow \infty} \int_{N}\left|\nabla u_{n}\right|^{p-2}\left\langle\nabla u_{n}, \nabla \phi\right\rangle d x \\
& =\int_{N} \lim _{n \rightarrow \infty}\left|\nabla u_{n}\right|^{p-2}\left\langle\nabla u_{n}, \nabla \phi\right\rangle d x \\
& =\int_{N}|\nabla u|^{p-2}\langle\nabla u, \nabla \phi\rangle d x=\int \phi d \mu
\end{aligned}
$$

where $N$ is some neighborhood containing $\partial D$ and $\partial D_{n}$ if $n$ is large enough and $\phi \in C_{0}^{\infty}(N)$. It follows that $\nu=\mu$ which is what we wanted to show. 


\section{References}

[1] Alessandrini, G., Lupo, D. And Rosset, E.: Local behavior and geometric properties of solutions to degenerate quasilinear elliptic equations in the plane. Appl. Anal. 50 (1993), no. 3-4, 191-215.

[2] Bennewitz, B. And Lewis, J.: On the dimension of $p$-harmonic measure. Ann. Acad. Sci. Fenn. Math. 30 (2005), no. 2, 459-505.

[3] Caffarelli, L., Fabes, E., Mortola, S. and Salsa, S.: Boundary behavior of nonnegative solutions of elliptic operators in divergence form. Indiana Univ. Math. J. 30 (1981), no. 4, 621-640.

[4] DiBenedetto, E.: $C^{1+\alpha}$ local regularity of weak solutions of degenerate elliptic equations. Nonlinear Anal. 7 (1983), no. 8, 827-850.

[5] Eremenko, A. And Lewis, J.: Uniform limits of certain $A$-harmonic functions with applications to quasiregular mappings. Ann. Acad. Sci. Fenn. Ser. A I Math. 16 (1991), no. 2, 361-375.

[6] Evans, L. C.: Partial Differential Equations. Graduate Studies in Mathematics 19. American Mathematical Society, Providence, RI, 1998.

[7] Federer, H.: Geometric Measure Theory. Die Grundlehren der mathematischen Wissenschaften 153. Springer-Verlag, New York, 1969.

[8] Gariepy, R. And Ziemer, W.: A regularity condition at the boundary for solutions of quasilinear elliptic equations. Arch. Rational Mech. Anal. 67 (1977), no. 1, 25-39.

[9] Gehring, F.: Uniform domains and the ubiquitous quasidisk. Jahresber. Deutsch. Math.-Verein. 89 (1987), no. 2, 88-103.

[10] Heinonen, J., Kilpeläinen, T. and Martio, O.: Nonlinear potential theory of degenerate elliptic equations. Oxford Mathematical Monographs. Oxford Science Publications. The Clarendon Press, Oxford University Press, New York, 1993.

[11] Henrot, A. and Shahgholian, H.: Existence of classical solutions to a free boundary problem for the $p$ Laplace operator: (I) the exterior convex case. J. Reine Angew. Math. 521 (2000), 85-97.

[12] Imaniec, T. And Manfredi, J.: Regularity of $p$ harmonic functions on the plane. Rev. Mat. Iberoamericana 5 (1989), no. 1-2, 1-19.

[13] Ladyshenskaya, O. and Ural'tseva, N.: Linear and quasilinear elliptic equations. Academic Press, New York-London, 1968.

[14] LEwIS, J.: Regularity of the derivatives of solutions to certain degenerate elliptic equations. Indiana Univ. Math. J. 32 (1983), no. 6, 849-858.

[15] Lewis, J.: Approximations of Sobolev functions in Jordan domains. Ark. Mat. 25 (1987), no. 2, 255-264.

[16] Lewis, J. And Vogel, A.: On pseudospheres. Rev. Mat. Iberoamericana 7 (1991), no. 1, 25-54. 
[17] Lewis, J. And Vogel, A.: Uniqueness in a free boundary problem. Comm. Partial Differential Equations 31 (2006), no. 10-12, 1591-1614.

[18] Gilbarg, D. And Trudinger, N.: Elliptic Partial Differential Equations of Second Order. Classics in Mathematics. Springer-Verlag, Berlin, 2001.

[19] Reshetnyak, Y.: Space Mappings with Bounded Distortion. Translations of Mathematical Monographs 73. American Mathematical Society, Providence, RI, 1989.

[20] Serrin, J.: Local behavior of solutions of quasi-linear equations. Acta Math. 111 (1964), 247-302.

[21] TolksdorfF, P.: Everywhere-regularity for some quasilinear systems with lack of ellipticity. Ann. Math. Pura Appl. (4) 134 (1983), 241-266.

Recibido: 7 de noviembre de 2006

Björn Bennewitz

Department of Mathematics and Statistics

University of Jyväskylä

FI-40014, Finland bennew@maths.jyu.fi

This work is the thesis of the author written under the direction of J. Lewis. 